\section{UJMM

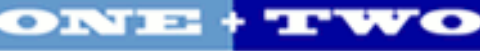

Volume 4 | 2012 Spring

\section{Undergraduate Journal of Mathematical}

Modeling: One + Two

2012

\title{
Complexity of Mitochondrial Genome Sequences
}

Brandon Toun

University of South Florida

\author{
Advisors: \\ Arcadii Grinshpan, Mathematics and Statistics \\ Egor Dolzhenko, Princeton University: Evolutionary Biology VSRC \\ Problem Suggested By: Egor Dolzhenko
}

Follow this and additional works at: https://digitalcommons.usf.edu/ujmm

Part of the Mathematics Commons

UJMM is an open access journal, free to authors and readers, and relies on your support:

Donate Now

\section{Recommended Citation}

Toun, Brandon (2012) "Complexity of Mitochondrial Genome Sequences," Undergraduate Journal of Mathematical Modeling: One + Two: Vol. 4: Iss. 2, Article 3.

DOI: http://dx.doi.org/10.5038/2326-3652.4.2.3

Available at: https://digitalcommons.usf.edu/ujmm/vol4/iss2/3 


\title{
Complexity of Mitochondrial Genome Sequences
}

\author{
Abstract \\ as another complexity measure of nucleotide sequences.

\section{Keywords} \\ Mitochondrial Genome, LZ77, Huffman Coding, Deflater Algorithm \\ Creative Commons License \\ (c) (i) ()
}

The purpose of this project is to compare the complexities of different species' mitochondrial genome sequences. Using an implementation of Deflate compression algorithm from Java standard library, we were able to compress mitochondrial genomes of nine different species. The complexity of each sequence is estimated as a ratio of the original sequence length to the length of the compressed sequence. In addition, we show how a notion of topological entropy from symbolic dynamics can be used

This work is licensed under a Creative Commons Attribution-Noncommercial-Share Alike 4.0 License. 


\section{TABLE OF CONTENTS}

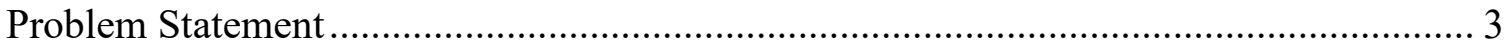

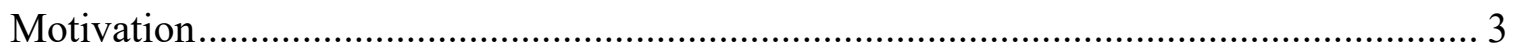

Mathematical Description and Solution Approach ................................................. 3

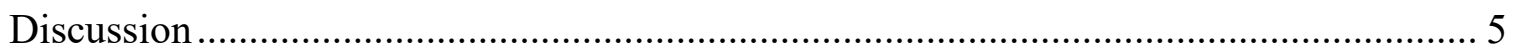

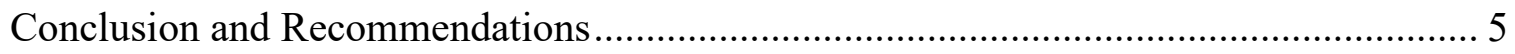

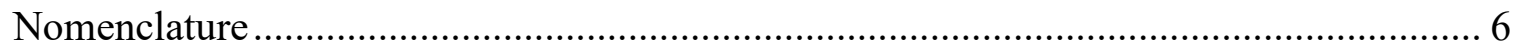

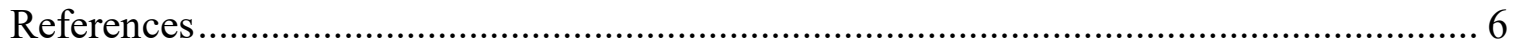

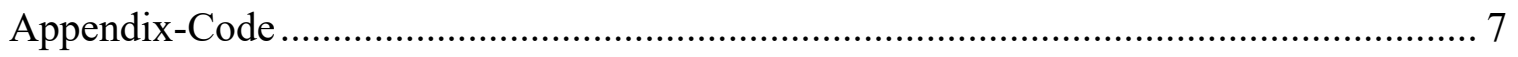

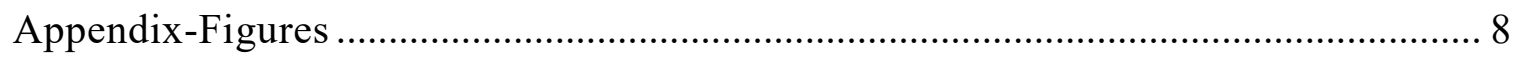

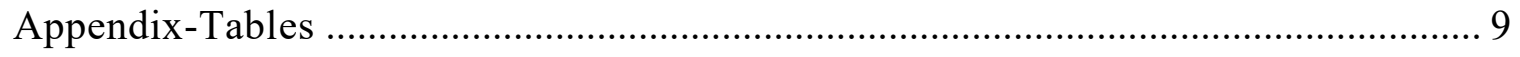




\section{PROBLEM STATEMENT}

Compare complexities of different species' mitochondrial genome sequences.

\section{MOTIVATION}

Any abnormalities in mitochondrial genome can be deleterious for the rest of the cell. In particular, various mutations in mitochondrial genomes are associated with human diseases (Ruiz-Pesini, Lott and Procaccio). Furthermore, it is conceivable that in a near future analysis of mitochondrial genomes becomes a routine way to diagnose such diseases. This calls for novel computational techniques for studying nucleotide sequences. In particular, comparing sequence complexity could be used to detect abnormalities in the mitochondrial genomes and can also be used to compare the genomes on an evolutionary scale.

\section{MATHEMATICAL DESCRIPTION AND SOLUTION APPROACH}

We are interested in comparing complexities of mitochondrial genome sequences of different species. Using sequences from a genomics database and a Java program to compress them we were able to estimate the complexity of these sequences (see Appendix A). We observed that the code works well even for large $(6-38 \mathrm{~kb})$ sequences.

The program prompts the user to enter a sequence and subsequently calculates the sequence length. It then compresses the sequence using the Deflater algorithm (Rose India Technologies; Feldspar), which is a part of the Java standard library.

The Deflater algorithm is a combination of Huffman coding (Huffman) and LZ77 (Ziv and Lempel) compression. Huffman coding gives each distinct value in a piece of data a code. 
For example, the word $w=A A C C C T T T T G G G G G$ consists of four distinct letters: $A, C, T$, and $\mathrm{G}$ that can be encoded by strings $1101,000,010,111$, respectively. Which implies that the word w can be encoded using $2 \times 4+3 \times 3+4 \times 3+5 \times 3=44$ whereas with 8 bit ASCII encoding for each symbol, $w$ requires $16 \times 8=128$ bits.

LZ77 algorithm encodes strings as a sequence of triples. For example, a string $w=$ ACT ACT $G$ is encoded by triples $(0,0, A),(0,0, C),(0,0, T),(3,3, G)$. The string $w$ can be reconstructed from these triples as follows: (1) first three triples mean that $A, C$, and $T$ must be appended to a word to get $A C T$, then (2) triple $(3,3, G)$ instructs to first add tree symbols starting at position 3 (from right to left) and then append a symbol $G$. The result is the original word $w$. It's clear that repetitive sequences can be encoded by this algorithm very efficiently.

The complexity is calculated according to the following expression

$$
C(w)=\frac{|w|}{\left|w_{i}\right|}
$$

Where $C(w)$ is the complexity of the sequence, $|w|$ is the length of the original sequence, and $\left|w_{i}\right|$ is the length of the sequence after compression. Note that $C(w)$ is never less than 1 ; the higher $C(w)$ is, the less complex the sequence is and vice versa.

Since DNA sequences are often extremely large, they could be studied with tools developed for infinite sequences in symbolic dynamics. One such tool is the topological entropy (Lind and Marcus) which could be calculated through the following the limit

$$
E(w)=\lim _{n \rightarrow \infty} \frac{1}{n} \log \left(F_{n}\right) .
$$

Here, $F_{n}$ stands for the number of all subsequences of $w$ of length $n$. For example, if $w=$ $A B C D E$, then $F_{1}=5$ since $A, B, C, D, E$ are exactly the subsequences of $w$ of length 1 . Similar 
calculation shows that $F_{2}=4$, and so on.

Although the entropy is defined for infinite sequences, you can still apply this notion to extremely long finite sequences, by calculating $F_{n}$ up to some fixed parameter $k$ that would depend of the length of the original sequence, or extending the original finite sequence into an infinite sequence by assuming that the extended sequence inherits the statistical properties of the original. In future, it would be interesting to develop the notion of topological entropy for finite sequences and determine how it compares to other measures of complexity.

\section{DISCUSSION}

Our estimates of complexities for the mitochondrial genomes of 9 species are presented in Table 1 . Note that the complexity may be affected by the size of the original sequence. For example, Laminaria digitata (large brown algae) had the largest mitochondrial genome sequence of the nine species and its complexity turned out to be quite high. The next two largest sequences are of Lottia digitalis (a mollusk) and Leishmania tarentolae (a virus) respectively. These were the least complex of the nine species, possibly because their mitochondria need fewer genes to function. The smallest and the least compressible sequence was that of Elaeis guineensis (an oil palm).

\section{CONCLUSION AND RECOMMENDATIONS}

In the future, it may be possible to quickly and efficiently sequence mitochondrial genomes. Thus, if a patient is believed to be developing a disease associated with mitochondrial mutations, the doctor may test whether the patient's mitochondrial genome is different from the normal (reference) genome. One possible way of doing this is to compress the sequence and look 
for a difference in complexity. If the complexity difference is indeed found, more elaborate computational techniques (such as sequence alignment) could be used to find out what exactly what the differences are.

\section{NOMENCLATURE}

\begin{tabular}{ll}
\hline Symbol & Description \\
\hline$|\boldsymbol{W}|$ & length of the sequence $W$ \\
\hline $\boldsymbol{C}(\boldsymbol{w})$ & length of the sequence $W$ \\
\hline $\boldsymbol{F}_{\boldsymbol{n}}$ & Complexity of the sequence $w$ \\
\hline & number of all subsequences of $w$ of length $n$ \\
\hline &
\end{tabular}

Feldspar, Antaeus. An Explanation of the DEFLATE Algorithm. 23 August 1997. 1 May 2012 $<$ http://www.cs.ucdavis.edu/ martel/122a/deflate.html $>$.

Huffman, D.A. "A Method for the Construction of Minimum-Redundancy Codes." Proceedings of the I.R.E. (1952): 1098-1102.

Lind, Douglas A and Brian Marcus. An Introduction to Symbolic Dynamics and Coding. Cambridge: Cambridge University Press, 1995.

Rose India Technologies. String Compression Using Deflater Class in Java. n.d. 27 April 2012 $<$ http://www.roseindia.net/tutorial/java/corejava/zip/compression.html $>$.

Ruiz-Pesini, E., et al. "An enhanced MITOMAP with a global mtDNA mutational phylogeny." Nucleic Acids Research 35 (Database issue) (2007): D823-D828.

Schaffer, S W and M.-Saadeh Suleiman. Mitochondria: The Dynamic Organelle. New York: Springer, 2007.

Ziv, Jacob and Abraham Lempel. "A Universal Algorithm for Sequential Data Compression." IEEE Transactions on Information Theory, 23(3) (1977): 337-343. 


\section{APPENDIX-CODE}

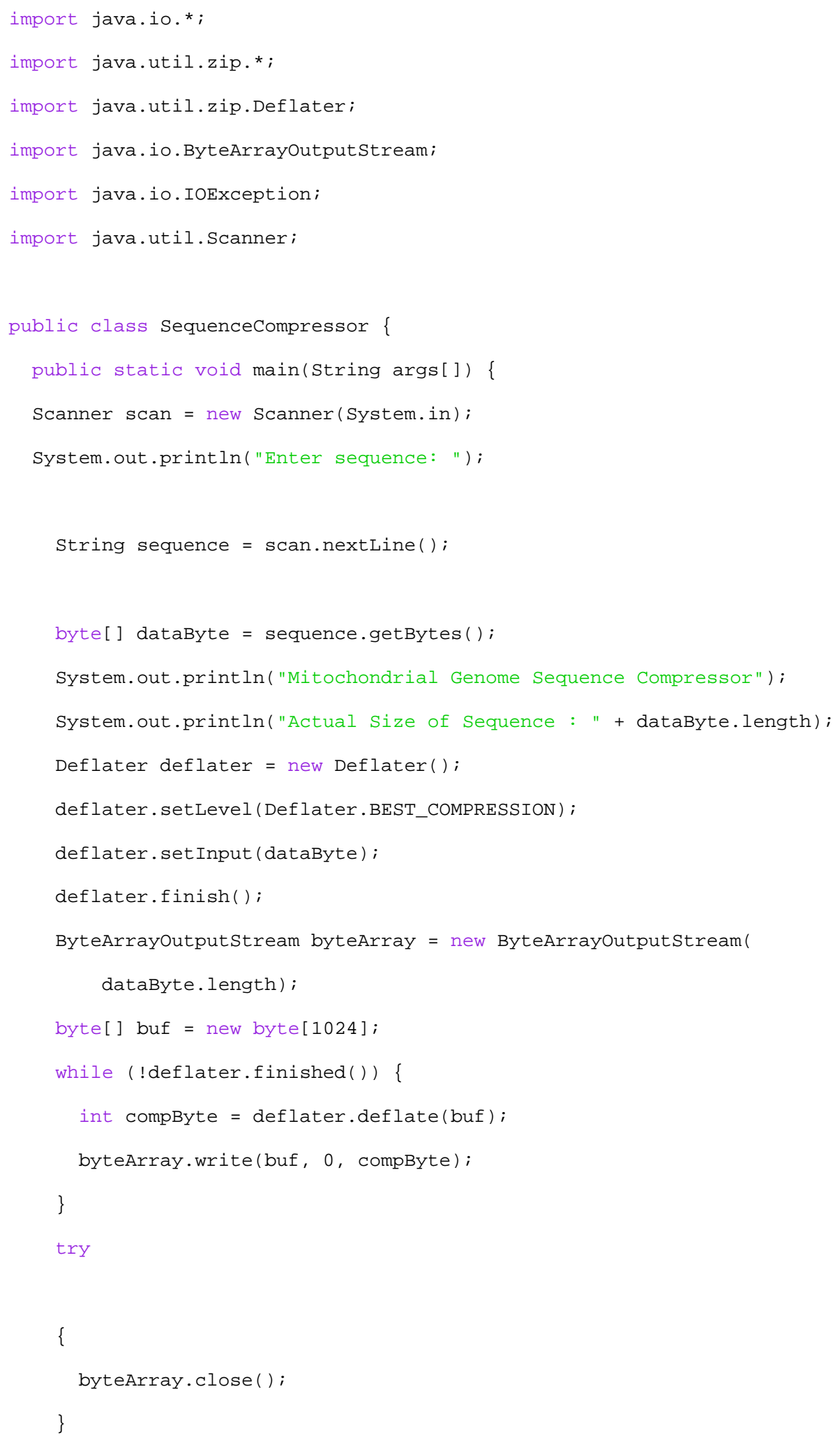




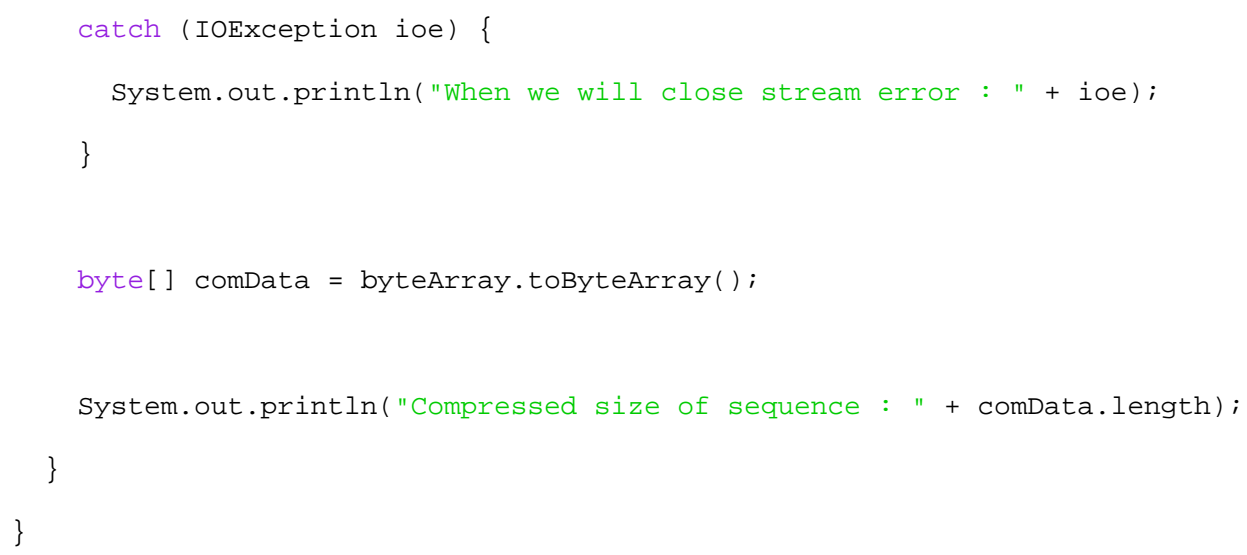

Listing 1. Java source code of the compression algorithm.

\section{APPENDIX-FIGURES}

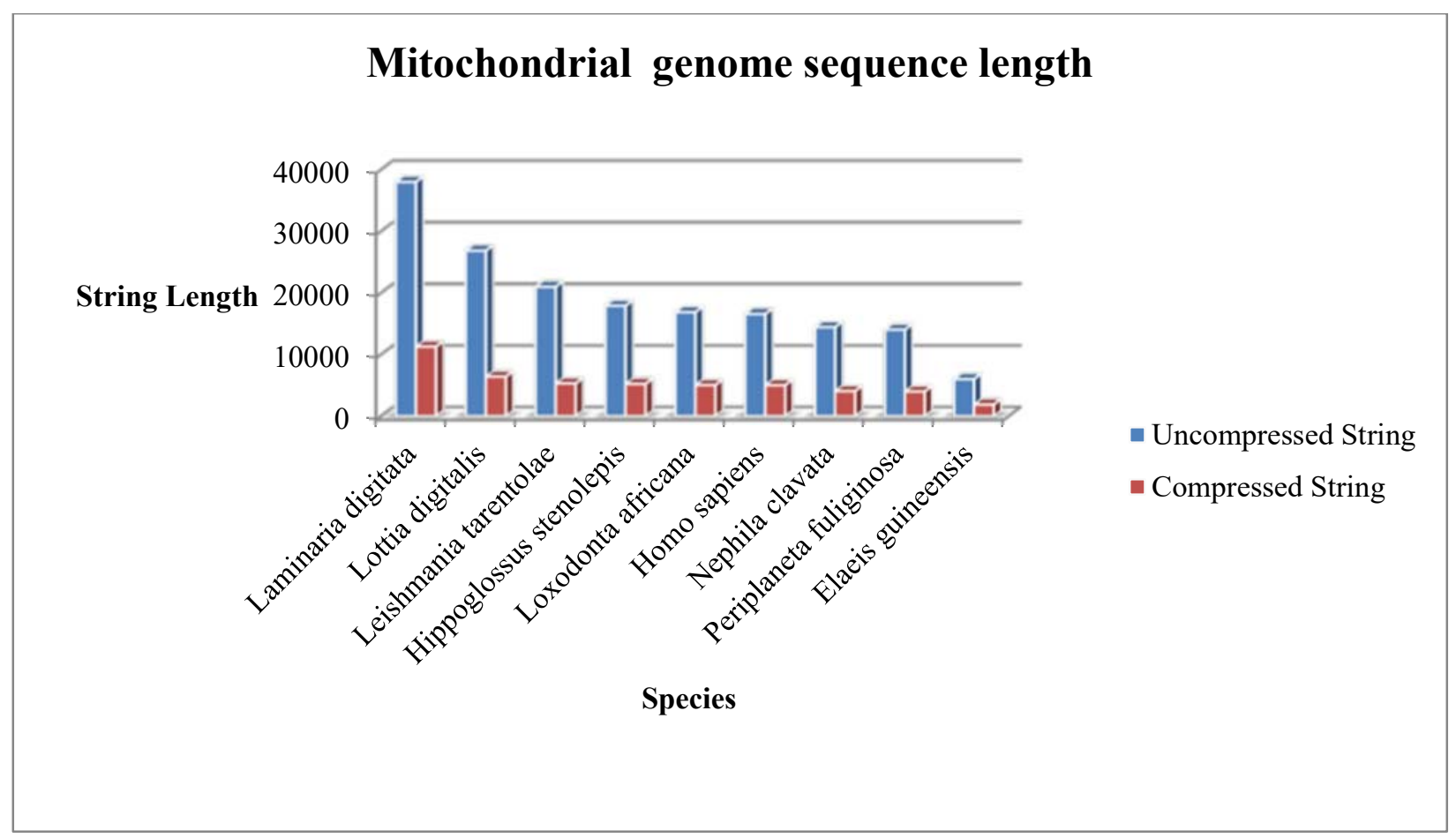

Figure 1: Bar graph comparing original sequence lengths to compressed versions. 


\section{Compression Coefficients}

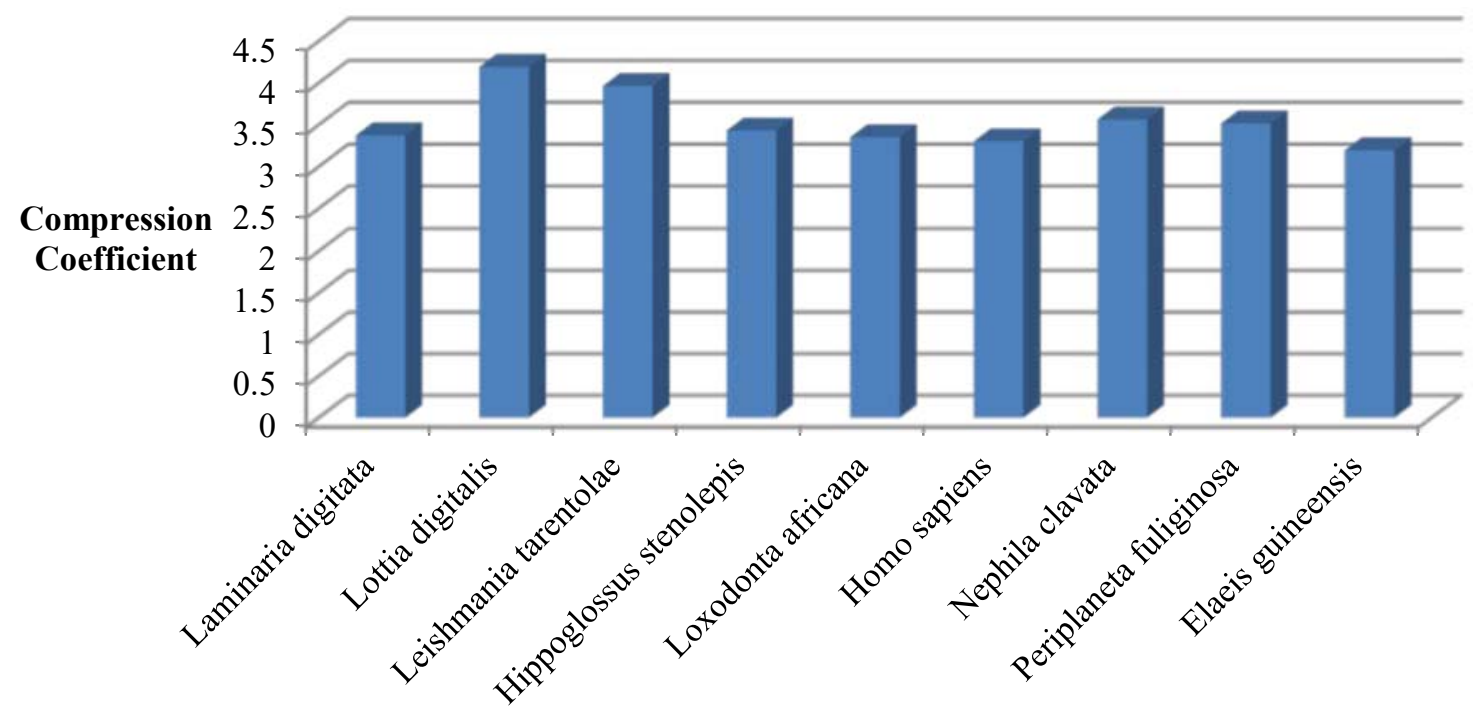

Species

Figure 2: Comparison of complexity of different species' mitochondrial genome sequences.

\section{APPENDIX-TABLES}

\begin{tabular}{|c|c|c|c|}
\hline Species & $\begin{array}{l}\text { Original Sequence } \\
\text { Length }\end{array}$ & $\begin{array}{c}\text { Compressed } \\
\text { Sequence Length }\end{array}$ & $\begin{array}{c}\text { Compression } \\
\text { Coefficient }\end{array}$ \\
\hline Laminaria digitata & 38007 & 11272 & 3.37 \\
\hline Lottia digitalis & 26835 & 6403 & 4.19 \\
\hline $\begin{array}{l}\text { Leishmania } \\
\text { tarentolae }\end{array}$ & 20992 & 5297 & 3.96 \\
\hline $\begin{array}{l}\text { Hippoglossus } \\
\text { stenolepis }\end{array}$ & 17902 & 5221 & 3.43 \\
\hline Loxodonta africana & 16866 & 5034 & 3.35 \\
\hline Homo sapiens & 16569 & 5019 & 3.30 \\
\hline Nephila clavata & 14436 & 4054 & 3.56 \\
\hline Periplaneta fuliginosa & 14021 & 3989 & 3.51 \\
\hline Elaeis guineensis & 6009 & 1881 & 3.19 \\
\hline
\end{tabular}

Table 1: Lengths of mitochondrial genomes and compression coefficients. 